\title{
The Double Slit Experiment Re-Explained
}

\author{
Mahmoud E. Yousif \\ Physics Department - The University of Nairobi P.O.Box 30197 Nairobi-Kenya
}

\begin{abstract}
The wavelet envisioned by Huygen's in diffraction phenomenon is re-interpreted as a Polarized Wave $(P W)$ after passing through slit/hole/or biaxial crystals which removed the electric field component from the Electromagnetic Radiation (EM-R), the resulted wave is what is known as the Conical Diffraction (CD) beam, the $\boldsymbol{P W}$ is originated from the Circular Magnetic Field $(\boldsymbol{C M F})$ produced by accelerated electrons, integrated with the Electric Field $(\boldsymbol{E F})$ during the Flip-Flop $(\boldsymbol{F}-\boldsymbol{F})$ mechanism producing EM-R; hence the passing of light through a single hole/slit/biaxial crystals, resulted in a $\boldsymbol{P W}$ which reproduced as rings on the monitor screen in single wave diffraction, while the interference of two such $\boldsymbol{P W}$ in double slits experiment, produced constructive or destructive interference forming patches on the monitor screen; and the perceived electron diffraction is an enter of two $\mathbf{C M F}$ from an accelerated electron into two slits then emerged to interfere constructively or destructively, and appears as patches, in addition to the electron which entered and emerged from the slit with the intense $\mathbf{C M F}$, the paper finally derived the origin of Planck' constant (h) for the second time; the logical interpretation of double slits diffraction will restore the common sense in the physical world, distorted by the pilot wave.
\end{abstract}

Keywords: Double Slit Experiment; wavelet; Circular Magnetic Field; electron diffraction; polarization; origin of Planck' constant.

\section{Introduction}

In his explanation to the photo electric effect in 1905, Einstein invoked quanta (photon) as theoretical justification to expel electron from the atom [1], which was viewed as a particle with zero rest mass [2], although the idea was rejected by many of his contemporary scientists lead by Millikan, [3], J.J. Thomson, Summerfield, and Richardson [4], but with endorsement from Compton experiment in 1922 [5], scientists gradually accepted the notion that electromagnetic radiation is a wave particle duality [6].

Contrary to light, where the discovery of diffraction preceded the wave theory, the electron diffraction was discovered as a consequence of a deliberate attempt to prove the wave nature of the electron [7], after de Broglie extended duality to particles in 1924 [8], then Davisson and Germer, explained the diffraction peak wave, generated by "electron wave," as the wavelength of Bragg formula, and resulted in diffraction pattern [9, 10], that was confirmed differently by G. P. Thomson [11]; although the reflected electrons from the nickel crystal in Davisson and Germer experiment, occurred before detection of diffracted beams [12], which posed contradiction on how deflected electron could stored reflected phantom wave? Regardless of that, both experiments became decisive in endorsing wave particle duality, making it acceptable, leading to new form of physics, contradicting the common sense and norm of life [13]. The ambiguity and uncertainty in this "electron wave," or "phantom wave" brought great confusions; which lead some to introduced the pilot wave, thought as similar to electromagnetic field [14], a state of confusion led Einstein to express at several occasions, before his death in 1955, that "for fifty years, he failed to understand what quanta (photon) is" [15] which amount to doubt on photon's existence, but the wave particle duality became acceptable by lack of sound alternative.

In the re-interpretation of Photoelectric Effects, it is suggested that, the Magnetic Radiation Force $\left(F_{m R}\right)$ is embedded in Electromagnetic Radiation (EM-R) [16], similar in nature to Planck' Radiation Energy $\left(E_{m R}\right)$ [17], while the production of Secondary Electromagnetic Radiation (S-EM-R) in "The Compton Effect Re-Visited" [18], consolidated the existence of an alternative interpretation base. This is based on exploring the characteristics of the Circular Magnetic Field $(\boldsymbol{C M F})$ produced by energetic electrons [19], and the Spinning Magnetic Force $(\mathrm{SMFc})$ produced by Spinning Magnetic Field (SMF), [20], it helped elaborating many phenomena, and subjected the double slit experiment into new analysis.

This paper is a modified version of an earlier version [21], it was fifth among series intended to prove the correctness of our "The Magnetic Interaction" [19] during a discussion [22], and since light represents more subtle and elusive problems than most other aspects of physical experience [23], and J. J. Thomson realized that the detection of a train of waves associated with the movement of electrons was not predicted by Maxwell's equations, emphasizing that, such a view of the electron had to be wrong [24], thus the existence of CMF produced by electron, not predicted by Maxwell's equation cast doubt about the acceleration mechanism for EM-R generation, and gives weight to the Flip-Flop (F-F) mechanism describing the transverse light wave mechanism [25], it helped explored and elaborated radiation energy, the conditions initiating EM-R generation 
and the nature and characteristics of Planck' Constant $(h)$ [26], it explained the Radiation Magnetic Force $\left(F_{m R}\right)$ embedded in EM-R, a phenomenon puzzled Einstein for fifty years [15], while the Planck' was explained as a parameter of constant element within the energy transformation process [16], and the Compton Effect was interpreted as a production of Secondary Electromagnetic Radiation (S-EM-R) phenomenon [18], all these prepared the ground for the existence of an alternative interpretation in micro-physical world, based on wave matter interactions.

Therefore, the diffraction of ripples through a single narrow opening, and two narrow openings [23], which was the bases for Huygen's principle of diffraction, explained with semi circle water waves generated in pond [27], is reinterpreted as the Conical Diffraction (CD) predicted theoretically by Hamilton in 1832 and observed the following year by Lloyed [28], the CD is formed from the Electromagnetic Radiation (EM-R), it's the Polarized Wave (PW), resulted when EM-R passed through a small hole/slit/biaxial crystals [29], this removed the electric field quantity, leaving the magnetic field, hence diffraction is interpreted as the resulted PW which neither travel with speed of light, nor carrying electric field, the PW is not semi-circle, rather it is a full circle, and composed of the magnetic portion of EM-R, it is the Circular Magnetic Field (CMF), which originated in the Flip-Flop mechanism during EM-R production [25], when passed through single slit, the PW appeared as single CD on the monitoring screen, while PW from two slits interfere constructively or destructively, and produced patches.

Since Electron diffraction is the strongest evidence for the principles of wave-mechanics on which the whole of atomic physics is based [30], hence relating the CMF and EM-R characteristics to Young's double slit experiment, and the reinterpretation of wavelets as $\mathrm{CMF}$, then formed in single slit diffraction as $\mathrm{PW}$, and the interference of two such PW in double slits experiment, resulted in constrictive or destructive interference; while electron diffraction is interpreted as the interference of two CMF entered and emerged from two slits and originated from a single accelerated electron, which accompanied the strongest CMF through one of the two slits, the paper finally derived the origin and structures of Planck' constant $(h)$ for the second time [16].

As the understanding of the dynamical processes in chemistry, materials science and biology on micro scale stems almost exclusively from time-resolved spectroscopy [31] which emerged from the diffraction carried by Bragg and based on Huygen's idea [32], therefore elaborating the true mechanism of this field in this and coming stage [33], would enrich and expand human understanding and unified the general level of human vision. In tackling this, it is better remember that, till early fifteen centaury, the Geocentric Model of the universe [34] was believed to reflect the true reality of the celestial bodies, forming an enforced doctrine, no lesson learned; and for nearly a century the duality dogma rejected all attempts to correct its oddness which diverted the general scientific mentality from the common sense pattern, thus limiting the scope of human endeavor.

\section{Energetic Circular Magnetic Field (CMF)}

The Circular Magnetic Field (CMF) produced by electrons in conductor carrying electric current originated from the magnetic phenomenon discovered by Hans Christian Oersted in 1819 [35], after which André Ampère determined the circular nature of the magnetic field around the conductor, and derived related formula using electric/magnetic parameters for a force between two conductors carrying electric current [36], and emphasis was directed towards electric field [37], thus the outweigh of electric parameters demised the CMF, where Maxwell equations in unified electric and magnetic fields, claimed both quantities equally contribute to the total energy density of the Electromagnetic Wave (EM-Wave) [38], but how this could be true when electric field in electron is not variable, while the magnetic field component designated as Circular Magnetic Field (CMF) increased with velocity or energy [39, 40, 41], and given as

$$
B_{C M F}=\frac{q V_{e}}{r_{m}^{2} c} T
$$

Where, $r_{m}$ is the magnetic radius in meter, $c$ is the velocity of light in $\mathrm{m} . \mathrm{s}^{-1}, V_{e}$ is the electron velocity in m.s ${ }^{-1}$, and $B_{C M F}$ is the $\boldsymbol{C M F}\left(B_{2 e}\right)$ in T.

The energy of Electromagnetic Radiation (EM-R) is shown to concentrate in the $\boldsymbol{C M F}\left(B_{C M F}\right)$ [26], this is why the magnitude of $\boldsymbol{C M F}\left(B_{C M F}\right)$ energy in Electromagnetic Radiation (EM-R) increased rapidly with frequency, because frequency is part of $\boldsymbol{C M F}$ formation [25] given as

$$
E_{R}=h v=\left\{\frac{B_{C M F}^{2} m_{e} c^{6}}{2\left(4^{4}\right) q^{2} v^{4}}\right\}=\left\{\frac{B_{C M F}^{2} 5.0527252584917691102101768251341 \times 10^{55}}{v^{4}}\right\}
$$

Where, $v$ is the Electromagnetic Radiation Frequency in $\mathrm{Hz}, m_{e}$ is electron mass in $\mathrm{kg}, q$ is charge in Coulomb, $h$ is Planck' constant in J.s., and the Radiation Energy $E_{R}$ is in Joules.

Table.1 give the variation of radiated energy with the $\boldsymbol{C M F}\left(B_{C M F}\right)$; using Eq. (2) or Eq. (21), this variation is also showed in the Secondary Electromagnetic Radiation (S-EM-R) [18], in which energetic $\boldsymbol{C M F}$ $\left(B_{C M F}\right)$ interacted with strong Nucleus Spinning Magnetic Field $\left(B_{1 U}\right)$ [18] producing EM-R [26], both 
magnitudes of the $\boldsymbol{C M F}$ and the Nucleus Spinning Magnetic Field $\left(B_{1 U}\right)$ are given in Table. 1, the $B_{1 U}$ is derived by [26]

$$
B_{1 U}=\frac{4 \pi m v_{F}}{q}=7.1447751068120606978208204881839 \times 10^{-11} v_{F} \quad T
$$

Where, $v_{F}$ is Flipping Frequency $\left(v_{F}\right)$ or radiation frequency $(v)$ in Hertz, and $B_{1 U}$ is the nucleus Spinning Magnetic Field (or the strong field). Substituting the equivalent of $v$ in Eq. (2) with $v$ in Eq. (3), the frequency is given by

Or as

$$
v=\sqrt[4]{\frac{2 \pi B_{C M F}^{2} m_{e}^{2} c^{6}}{\left(4^{4}\right) B_{1 U} q^{3} h}} H z
$$

$v=\sqrt[4]{\frac{5.4482605349776842482155769903031 \times 10^{78} B_{C M F}^{2}}{B_{1 U}}} \quad \mathrm{~Hz}$

Therefore, and as given by Eq. (4), the radiation energy and frequency for each $\boldsymbol{C M F}\left(B_{C M F}\right)$ given in Table.1 is related to the magnitude of Nucleus Spinning Magnetic Field $\left(B_{1 U}\right)$ which trigger it [18] as given by Eq.(3), the same is the conclusion of high energy scientists, that the shortest radiation/particle bursts of x-rays and $\gamma$-rays are produced by the highest power laser [42], hence substuting $v$ in Eq. (3) with $v$ in Eq. (4) the relation between

\begin{tabular}{|c|c|c|c|c|c|c|c|}
\hline$B_{1 U}=\sqrt[5]{1}$ & 41975 & 9394711 & 25686987081 & 731 & $10^{38} B_{C M}^{2}$ & $T$ & (6) \\
\hline$V_{e}\left(m s^{-l}\right)$ & $E_{R}(J)$ & $v(H z)$ & $t_{p}(s)$ & $\gamma(\mathrm{m})$ & $\begin{array}{c}r_{m}(N / 4) \\
(m)\end{array}$ & $\begin{array}{c}B_{G M F} \\
(T)\end{array}$ & $\bar{B}_{I t}(T)$ \\
\hline $\begin{array}{c}1.2061422956252 \\
77935879782301 \\
1235 \times 10^{5}\end{array}$ & $\begin{array}{l}6.62607 \\
55 \times \\
10^{-21}\end{array}$ & $1.0 \times 10^{13}$ & $1 . \times 10^{-13}$ & $\begin{array}{l}3, \times \\
10^{-5}\end{array}$ & $\begin{array}{l}7.5 x \\
10^{-6}\end{array}$ & $\begin{array}{c}1.145157832773320 \\
5830019441826343 \\
\times 10^{-12}\end{array}$ & $\begin{array}{c}7.144775106812060 \\
6978208204881839 \\
\times 10^{2}\end{array}$ \\
\hline $\begin{array}{c}3.8141568364400 \\
21581956544294 \\
8061 \times 10^{6}\end{array}$ & $\begin{array}{c}6.62607 \\
55 \times \\
10^{-18}\end{array}$ & $1.0 \times 10^{16}$ & $1 . \times 10^{-16}$ & $\begin{array}{l}3 . x \\
10^{-8}\end{array}$ & $7.5 e-9$ & $\begin{array}{c}3.621307031945908 \\
4345608961862395 \\
\times 10^{-5}\end{array}$ & $\begin{array}{c}7.144775106812060 \\
6978208204881839 \\
\times 10^{5}\end{array}$ \\
\hline $\begin{array}{c}1.2061422956252 \\
779358797823011 \\
235 \times 10^{7}\end{array}$ & $\begin{array}{l}6.6260 \\
755 \times \\
10^{-17}\end{array}$ & $1.0 \times 10^{17}$ & $1 . \times 10^{-17}$ & $\begin{array}{l}3, x \\
10^{-9}\end{array}$ & $\begin{array}{l}7.5 \times \\
10^{-10}\end{array}$ & $\begin{array}{c}1.145157832773320 \\
5830019441826343 \\
\times 10^{-2}\end{array}$ & $\begin{array}{c}7.144775106812060 \\
6978208204881839 \\
\times 10^{6}\end{array}$ \\
\hline $\begin{array}{c}1.2061422956252 \\
779358797823011 \\
235 \times 10^{3} \\
\end{array}$ & $\begin{array}{c}6.62607 \\
55 \times \\
10^{-15}\end{array}$ & $1.0 \times 10^{19}$ & $1 . \times 10^{-19}$ & $\begin{array}{c}3 . \times \\
10^{-11}\end{array}$ & $\begin{array}{r}7,3 x \\
10^{-12}\end{array}$ & $\begin{array}{c}1.145157832773320 \\
5830019441826343 \\
\times 10^{3} \\
\end{array}$ & $\begin{array}{c}7.144775106812060 \\
6978208204881839 \\
\times 10^{8} \\
\end{array}$ \\
\hline $\begin{array}{c}6.6063134287502 \\
936063284554884 \\
65 \times 10^{9}\end{array}$ & $\begin{array}{l}1.98782 \\
265 \times \\
10^{-11}\end{array}$ & $3 . \times 10^{22}$ & $\begin{array}{l}3.333333333333 \\
3333333333333 \\
333333 \times 10^{-23}\end{array}$ & $\begin{array}{c}1 . x \\
10^{-14}\end{array}$ & $\begin{array}{l}2.5 x \\
10^{-15}\end{array}$ & $\begin{array}{c}5.645058992222308 \\
8345015144489350 \\
8 \times 10^{11}\end{array}$ & $\begin{array}{c}2.14343253204361 \\
8209346246146455 \\
2 \times 10^{12}\end{array}$ \\
\hline$V_{e}\left(m s^{-l}\right)$ & $E_{R}(J)$ & $f(H z)$ & $t_{F}(s)$ & $\lambda(\mathrm{m})$ & $\begin{array}{c}r_{m}(N / 4) \\
(m)\end{array}$ & $\begin{array}{c}B_{G M F} \\
(T)\end{array}$ & $\begin{array}{l}B_{I t} \\
(T)\end{array}$ \\
\hline
\end{tabular}
$B_{C M F}$ and $B_{1 U}$ is given by [26]

Table.1. The Circular Magnetic Field $\left(B_{C M F}\right)$ produced by an accelerated electron represents Electromagnetic Radiation Energy $\left(E_{R}\right)$, carried by wave of specific frequency. The Table shows from left, electron's velocity $\left(V_{e}\right)$, radiation energy $\left(E_{R}\right)$, frequency $(v)$, Flipping Time $\left(t_{F}\right)$, wavelength $(\lambda)$, the magnetic Radius $\left(r_{m}=\lambda / 4\right)$, $\boldsymbol{C M F}\left(B_{C M F}\right)$, and the Nucleus Spinning Magnetic Field $\left(\boldsymbol{B}_{I U}\right)$, which triggers the release of the radiation [18].

As the $\boldsymbol{C M F}$ is resulted from an accelerated charge as given by Eq. (1), hence this equation can derive the magnetic field of fast moving nuclei with electric charges in Relativistic Heavy Ion Collider (RHIC) and Large Hadrons Collider (LHC), it created extreem strongest magnetic fields [43], given in Table.2, and compared with varrities of magnetic fields; this is also to be compared with the $\boldsymbol{C M F}$ in Table.1; the $\boldsymbol{C M F}$ at frequency of 3. $\times 10^{22} \mathrm{~Hz}$ in Table.1, contained 5.6450589922230883450151444893508 $\times 10^{11}$ Tesla, and required nucleus strong field of $2.1434325320436182093462461464552 \times 10^{12}$ Tesla to trigger it and pull the radiated $E M-R$ [18] after the end of $\boldsymbol{F}-\boldsymbol{F}$ mechanism [26].

\begin{tabular}{|l|c|}
\hline \multicolumn{1}{|c|}{ The source (Realization as) } & Strength Tesla \\
\hline Earth's magnetic field & $6 . \times 10^{-5}$ \\
\hline A typical hand-held magnet & $1 . \times 10^{-2}$ \\
\hline Superconducting magnets in LHC & $8.3 \times 10^{0}$ \\
\hline Strongest steady magnetic field & $4.5 \times 10^{1}$ \\
\hline Surface field of neutron stars & $\sim 1 . \times 10^{8}$ \\
\hline Critical magnetic field of electrons & $4 . \times 10^{9}$ \\
\hline Surface field of magnetars & $\sim 1 . \times 10^{11}$ \\
\hline Noncentral heavy-ion coll. at RHIC & $\sim 1 . \times 10^{13}$ \\
\hline Noncentral heavy-ion coll. at LHC & $\sim 1 . \times 10^{14}$ \\
\hline
\end{tabular}

Table2. Comparison of magnitudes of several sources of Magnetic fields [42] 


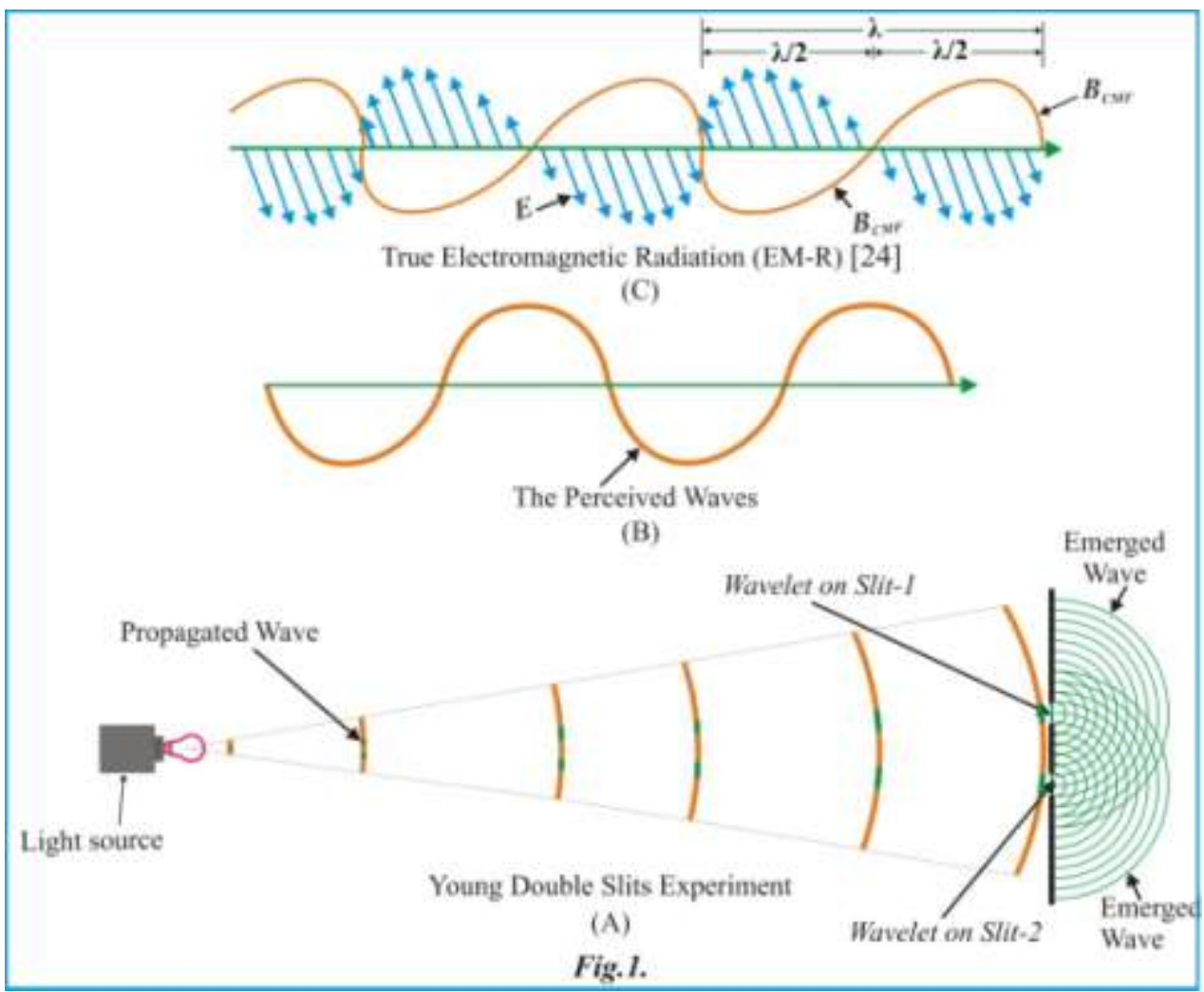

Fig.1. Young' Double Slits experiment showing in (A), the wavelet depicted to enters the slits, and acts as secondary sources [44], the perceived radiation wave is shown in (B), while (C) shows the true shape of Electromagnetic Wave $(\boldsymbol{E} \boldsymbol{M}-\boldsymbol{W})[25]$.

\section{Circular Magnetic Field (CMF) Wavelet}

Young's famous "double-slit experiment" provided convincing evidence that light does have properties explainable only in terms of waves [23], and the diffraction of ripples through a single and two narrow openings $[36,23]$, was the base behind Huygen's principle of diffraction, explained in connection with the semi circle water waves generated in pond [27], and the pattern resulted from the superposition of diffracted waves from both slits is interpreted as that produced by two point sources vibrating in phase [23]; but the produced circular wave train in water, and the overlapping interference by two ripple pattern produced by two vibrating points source in phase [23], clearly shows the circular nature of the produced waves, and the resulted interference produced a diffraction [44], therefore Young's double slits experiment shown in Fig.1-A, in which light is shown to propegate from the source to the two slits, where the propegated wave is alway percieved as consisting of sinsodial shape ilustrated in Fig.1-B with slits in turn produced wavelets, acting as secondary waves or sources of light according to Huygens' construction [44], while the practical requirements for narrow slits which are the source of just one Huygens' wavelet are difficult if not impossible to achieve [44], and since diffraction is "the pattern of beams which occur when light passes through pinholes and nets or is reflected from graduated rulers," [43] and the geometrical conditions for constructive/destructive interference which apply to just one wavetrain apply to all wavetrains, and the resulted patterns of light and dark on the screen is the diffraction pattern [44], therefore, these showed the diffraction been conceived from the geometrical perspective not the physical dynamics of the slits on the emerged waves in addition to wave structure, which complicated the single aperture pattern; therefore, re-studying the mechanism behind the entering and emergence of light from a hole/slit, suggested different mechanism; and since water wave composed of single wave, while Electromagnetic Wave (EM-W) consist of electric and magnetic fields [45], unfortunately the shape of this wave have been mixed with the semi circle water waves generated in pond, interpreted as synonymous to magnetic wave [27], as shown in Fig.1-A; but since it was discovered that, the electric field (E-F) produced in series of time-lapse photographs, is always either pointing up or down [38], similar to the circularly polarized light [46], and since both experiments showed electric field as raising and falling along the propogating path and interpreted as moving vertically, and since polarization of light is the filtering of one component of the incident beam [47], thus the perceived double slit shape in Fig.1-A, is neither the correct shape of EM-R entering the slit, nor the correct shape emerging from the slit, therefore the true shape of EM-R entering the slit is the one shown in Fig.1-C; hence the passing of EM-R through a slit/hole/biaxial crystals [29], remove its electric field due to 
polarization process, hence the slit as a filter component restrained the electric field, hence the wavelet entering the slit is the Circular Magnetic Field $(\boldsymbol{C M F})$ designated as $\boldsymbol{\delta}-\boldsymbol{C M F}$ shown in Fig.2-A, therefore the emerged wavelet is re-interpreted as a change in the EM-R, where the resulted wave neither travel with speed of light, nor carrying electric field, the wave is not semi-circle, rather it is a full circular wave, it's the Polarized Wave (PW), it composed of the magnetic part of the EM-R, this is what appears as the Conical Diffraction (CD) [28]; the PW originated from the Circular Magnetic Field ( $\boldsymbol{C M F}$ ) produced by accelerated electrons [25] as given by Eq.(1), and shown in Fig.2-B, therefore the $\boldsymbol{P W}$ (or $B_{P W}$ ) is the magnetic part of electromagnetic radiation contained the radiation energy given by Eq. (2) and shown in Table.1, hence from Eq. (2), the $\boldsymbol{P W}\left(B_{P W}\right)$ of this energy is given by

$$
B_{P W}=\sqrt{\frac{2\left(4^{4}\right) q^{2} v^{4} E_{R}}{m_{e} c^{6}}} \quad T
$$

Replacing $E_{R}$ in Eq. (7) with $v h$, hence $B_{P W}$ is given in terms of frequency as

$$
B_{P W}=\sqrt{\frac{2\left(4^{4}\right) q^{2} v^{5} h}{m_{e} c^{6}}}=\sqrt{1.3113864619620884691409896280354 \times 10^{-89} v^{5}} T
$$

The $\boldsymbol{P W}$ given by Eq. (8) is to be derived in term of frequency, the $B_{P W}$ is given by

$$
B_{P W}=\sqrt{C_{R} v^{5}} \quad T
$$

Where, $C_{R}$ is the constant of radiation it is equal to $1.3113864619620884691409896280354 \times 10^{-89} \mathrm{~T}^{2}$. $\mathrm{Hz}^{-5}$ $\left(\mathrm{T}^{2} \cdot \mathrm{s}^{5}\right)$.

The $\boldsymbol{P W}\left(B_{P W}\right.$ given by Eq. (9), is in terms of frequency, while the frequency $v$ in Eq. (8) can be written as

$$
v^{5}=\frac{E^{5}}{\mathrm{~h}^{5}}=\frac{B_{P W}^{2} m_{e} c^{6}}{2\left(4^{4}\right) q^{2} h}=\frac{m^{4} V_{e}^{8}}{2^{4} \mathrm{~h}^{4}}
$$

From Eq. (10) the following is derived

$$
V_{e}^{8}=\frac{2^{4} \mathrm{~h}^{4} B_{P W}^{2} c^{6}}{2\left(4^{4}\right) q^{2} E_{R} m^{3}}
$$

Since velocity is given in term of energy as

$$
V_{e}=\sqrt{\frac{2 \mathrm{E}}{m}} \quad \mathrm{~m} / \mathrm{s}
$$

For $V_{e}^{8}$ the above value is

$$
V_{e}^{8}=\frac{2^{4} \mathrm{E}^{4}}{m^{4}}
$$

Substituting the left hand side of Eq.(11) with the right hand side of Eq. (13)

$$
\frac{2^{4} E^{4}}{m^{4}}=\frac{2^{4} h^{4} B_{C M F}^{2} c^{6}}{2\left(4^{4}\right) q^{2} E_{R} m^{3}}
$$

Re-arranging Eq.(14), the $\boldsymbol{C M F}\left(B_{C M F}\right)$ is given by

$$
B_{C M F}=\sqrt{\frac{5.12 \times 10^{2} q^{2} \mathrm{E}^{5}}{\mathrm{~h}^{4} m c^{6}}} \quad T
$$

From Eq.(15), the $\boldsymbol{C M F}\left(B_{C M F}\right)$ can be given by

$$
B_{C M F}=\sqrt{1.0267123723266052069501087332373 \times 10^{77} \mathrm{E}^{5}} \quad T
$$

Therefore, from Eq. (15) the EM-R energy is given by

$$
E_{R}=\sqrt[5]{\frac{B_{C M F}^{2} \mathrm{~h}^{4} m c^{6}}{5.12 \times 10^{2} q^{2}}} \quad J
$$

Since the $\boldsymbol{C M F}\left(B_{C M F}\right)$ is the only variable in Eqs. (15\&17), therefore the EM-R energy in terms of the $\boldsymbol{C M F}$ $\left(B_{C M F}\right)$ is given by

$$
E_{R}=\sqrt[5]{\frac{B_{C M F}^{2}}{1.0267123723266052069501087332373 \times 10^{77}}} \quad J
$$

Therefore, the energy of the EM-R entering the slit in Fig.2-A, is contained in the $\boldsymbol{C M F}\left(B_{C M F}\right)$ as given by Eq. (18), and it's value can be derived using Eq. (16). 


\section{Young's Experiment And Resulted Polarized Wave (PW)}

The electromagnetic wavelet entered and emerged from a small hole comparable to its wavelength is shown in Fig.2-A; as explained above, this wavelet is the magnetic part of the radiation as given by Eq. (15), and since a wavelet emerging from a slits in turn act as secondary wave or source of light according to Huygens' [44], but as shown in the polarization process it's a source of wave, but not light, therefore any such $\boldsymbol{\delta}$-CMF emerging from a small hole or slit, is suggested to restored to its $\boldsymbol{C M F}$ origin and is a Polarized Wave (PW); therefore as shows in Fig.2-B, the transformation of the Circular Magnetic Field-Electric Field (CMF-EF) into Electromagnetic Radiation $(\boldsymbol{E M}-\boldsymbol{R})$ through the Flip-Flop $(\boldsymbol{F}-\boldsymbol{F})$ mechanism [25], is re-transformed into PW through the polarization mechanism when it passed through a hole (aperture)/slit/biaxial crystals, and appeared as a Conical Diffraction (CD) on the screen [29].

The change of the magnetic wavelet with dimension is synonymous to the restoration of the field into the PW (or $\boldsymbol{C M F}$ ), but without electric field, this is expressed by

$$
P W=(C M F+E F)-(E F) \quad T
$$

Since the $\boldsymbol{C M F}\left(B_{C M F}\right)$ is the only variable in the radiation energy given by Eq. (17), and the formula shows $\boldsymbol{C M F}\left(B_{C M F}\right)$ as the main energy in Electromagnetic Radiation (EM-R) shown in Fig.1-C [25], and as diffraction pattern from a single slit is a central maximum with much fainter bands of half the width of the central maximum on each side, and diffraction pattern from a circular hole or aperture is, correspondingly, a central disc surrounded by much fainter rings or haloes [44] shown in Fig.2-C, which are equivalent to the CD obtained while using biaxial crystals [28], therefore these characteristics imply on the resulted $\boldsymbol{P} \boldsymbol{W}$ showed in Fig.2-A, and resulted in the diffraction pattern shown in Fig.2-C [44], which is a representation of the $\boldsymbol{P W}\left(B_{P W}\right)$ as it emerged from aperture of Fig.2-A; while the Poisson's or Arago spot shown as a white spot in each of Fig.2-C circles, was interpreted as due to the existence of diffracted point sources at phase in the central axis, so the waves will add up and create a bright spot at the center of the image [48], rather this is suggested to be similar to the bright spot firstly observed by Raman [49], but as shown in Fig.2-C, this spot is part of the resulted $\boldsymbol{P} \boldsymbol{W}$ emerged from EM-R after the removal of the electric field, this becomes clear with distance [28], as discovered by Raman [49], it is in the position occupied by an electron before $\boldsymbol{C M F - E F}$ is formed as shown in Fig.2-B.

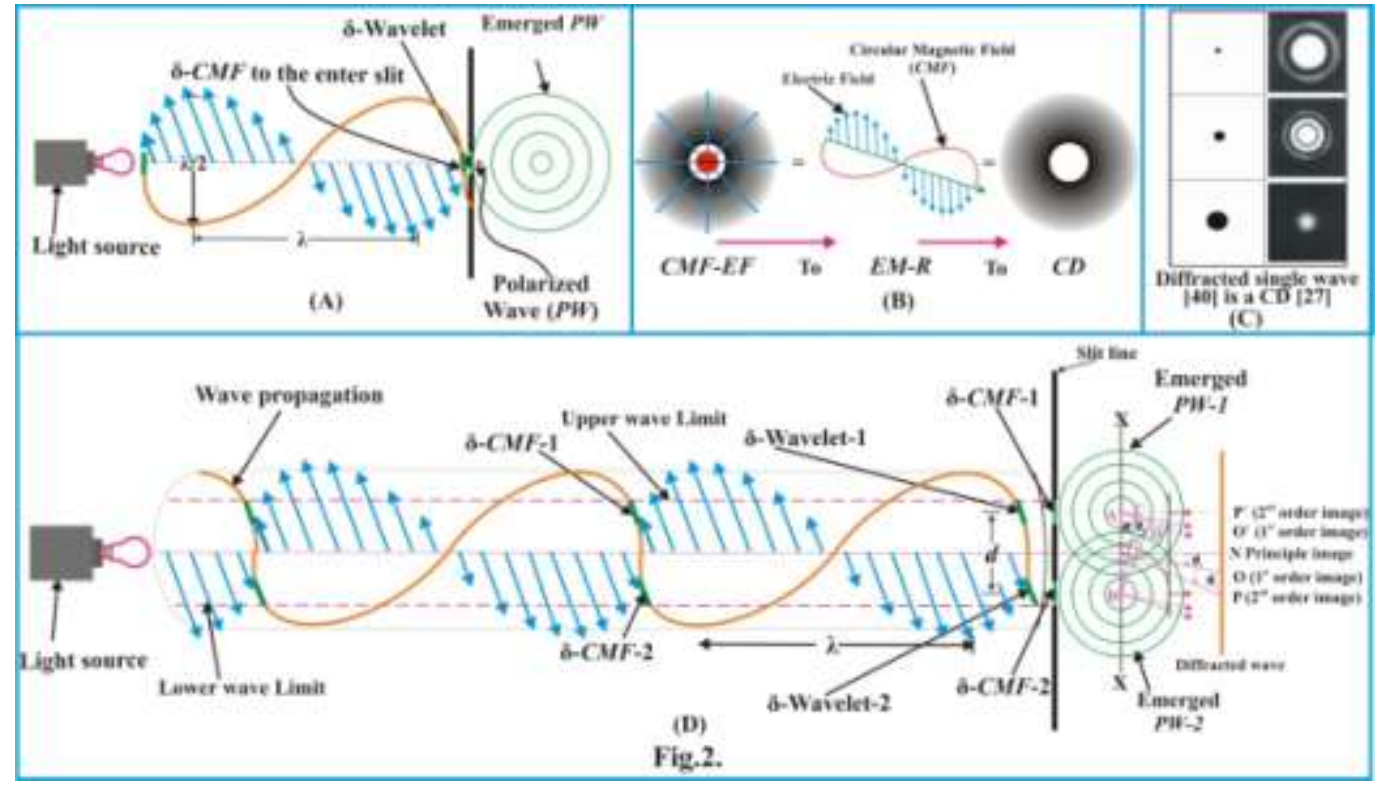

Fig.2. In (A) Electromagnetic Radiation (EM-R) emerged from single slit as a Polarized Wave (PW), while (B) shows the Flip-Flop $(\boldsymbol{F}-\boldsymbol{F})$ transformation of both $\boldsymbol{C M F}$-Electric Field $(\boldsymbol{C M F}-\boldsymbol{E F})$ forming $\boldsymbol{E M}-\boldsymbol{R}$ [25] then back to the polarized Conical Diffraction $(\boldsymbol{C D})$ on screen [28], (C) shows single diffracted or PW (CMF) from three holes with different sizes [44], it's shape is similar to $\boldsymbol{C D}$ [28], and (D) shows two $\boldsymbol{P W}$ resulted from wavelets $\boldsymbol{\delta}-\boldsymbol{C M F - 1}$ and $\boldsymbol{\delta}-\boldsymbol{C M F}-2$ of the EM-R, the geometrical structure of line $\mathrm{x}-\mathrm{x}$ is on right of the slit line, interference of both $\boldsymbol{P} \boldsymbol{W}$ produced diffracted patches.

The Young's double-slit experiment is basically, involves splitting a single beam of light into two beams in order to ensure that they are in phase, then allowed to overlap, and the two wave trains interfere, constructively in some places and destructively in others [23], the diffraction pattern is taken to be created by the interference of waves traversing two clearly separated paths [50], but as shown in Fig-2-A, the emerged polarized wave lost its electric field, it represents the $\boldsymbol{C D}$ shown in Fig.2-B\&C; therefore what really takes place 
in Young's double slit experiment shown in Fig.2-D, is that both $\delta-B_{C M F-1}$ and $\delta-B_{C M F-2}$, entered slit-1 and slit-2 respectively, they both transformed into $B_{P W-R 1}$ and $B_{P W-R 2}$ waves; therefore, the Young's doubleslit experiment is an interference carried by two intense Polarized Waves $(\boldsymbol{P} \boldsymbol{W})$ beams as shown in Fig.2-D, the magnitudes of these $\boldsymbol{P W}$ is as derived for the $\boldsymbol{C M F}$ in Table.1, and given by Eq. (9).

The $d$ in Fig.2-D, is the distance of diffraction grating, and since line $x-x$ is parallel to the slit line, therefore the geometrical conditions are constant for constructive/destructive interference which apply to both wavetrain and apply to all wavetrains, and the resulted pattern of light and dark patches on the screen is the diffraction pattern [44], and the formula for this is apply [36]

$$
\lambda=\frac{d \sin \theta_{n}}{n} \quad m
$$

Where, $d$ is the diffraction grating, $\lambda$ is the wavelength of the incident light, $\theta$ is the diffraction angle, and $n$ is the order of the image.

\section{Electron's Circular Magnetic Field (CMF) Diffraction}

It is known that, the phase waves or matter waves, exhibit certain striking points of similarity with electromagnetic waves, particularly in their ability to produce the diffraction effects by which they were discovered [12], and Thomson realized his experiment showed the central spot and rings were deflected together, and they are due to cathode rays of significantly the same velocity [11], this represents a simultaneous deflection characteristic, which is a hint for extraordinary conflicting situation; but not investigated, while G.P. Thomson questioned the nature of these waves? And relation it has with electron associated with it [23], these scientific concerns were not answered at time; our answer is given by Eq. (1), in which any accelerated charged produced Circular Magnetic Field $(\boldsymbol{C M F})$, which was perceived as the phase waves, matter waves [12] or pilot waves [14]; hence in double slit experiment shown in Fig.3, twenty seven electrons were accelerated and ejected by an electron gun, successively towards the two slits; the figure is divided into three sections; first the plan showing the electron gun with the last electron-27 emerging from the gun; the second part is two dimensional perspective of three electrons-26-25-24 surrounded along its trajectory by layers of Circular Magnetic Fields $\left(\boldsymbol{C M F}\right.$ or $\left.B_{C M F}\right)$ of varied magnitudes with each moving towards one of the two slits; the third part is a plan shows electron-23 before entering slit-2 it's $\boldsymbol{C M F}$ is marked with green color, electron-22 is in the center of slit1 surrounded by $\delta-B_{C M F-1}$, while part of the $\boldsymbol{C M F - 2 2}$ or the $\delta-B_{C M F-2}$ (with lower magnitude ranging between $0.25 \%$ to $56.25 \%$ of main $\boldsymbol{C M F}$ ) showed in center of slit-2, then electrons-21 just exited from slit-2, while electron-20 before it and both impinged on the monitor, which showed resulted five bright fringes or patches resulted from previous nineteen interferences (19) $\mathrm{x}\left(2 \delta-B_{C M F}\right)$ of constructive waves that had emerged from both slits.

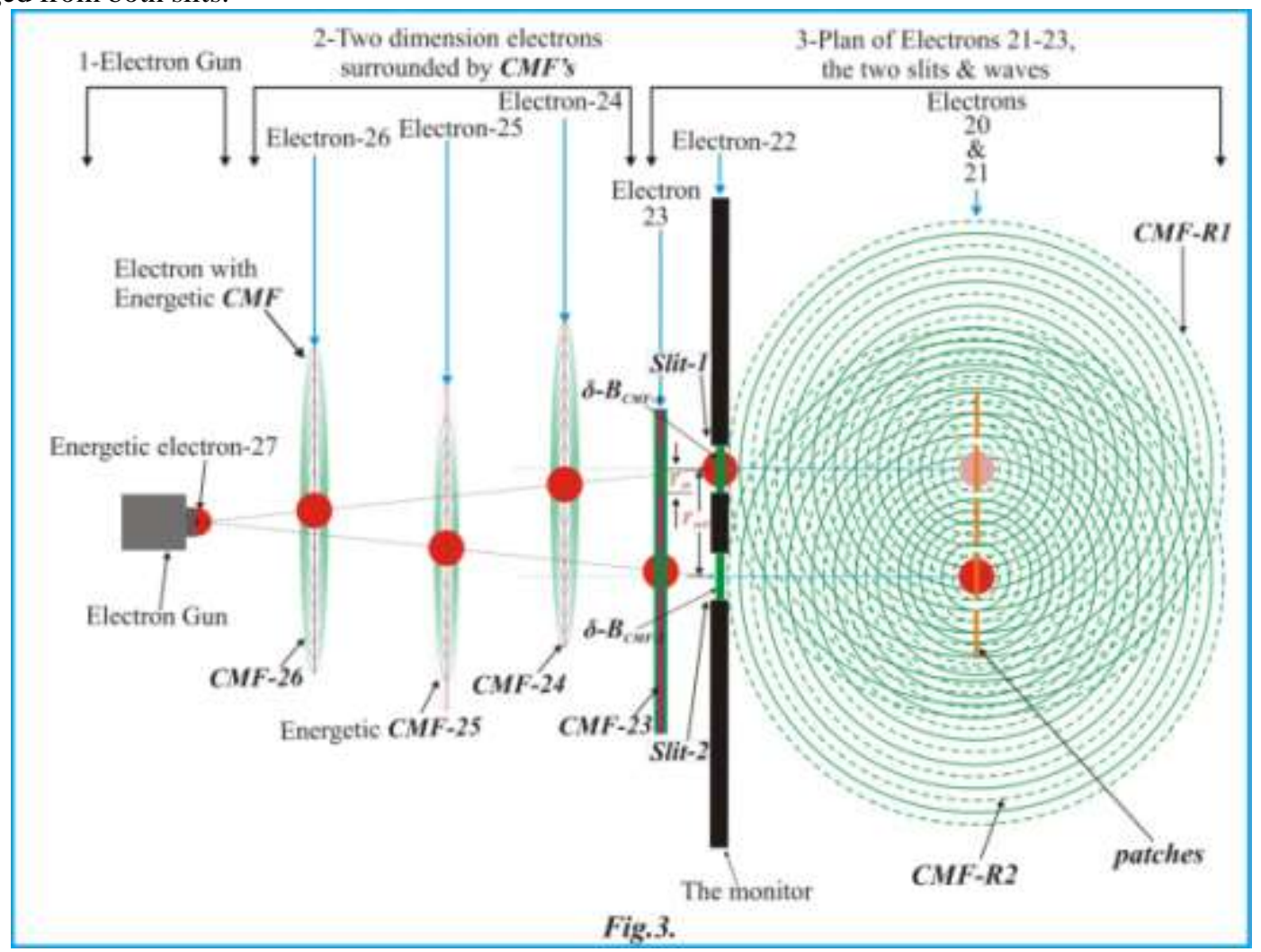


Fig.3. The diffraction of the Circular Magnetic Field (CMF), produced by an electron. Section 1- shows the last electron 27 emerging from electron gun, 2-two dimensions of three electrons moving towards the two slits surrounded by $\boldsymbol{C M F}$ and $\boldsymbol{E F}, 3$-shows electron-23 before entering the second slit surrounded by $\boldsymbol{C M F}$, then electronn-22 with $\boldsymbol{\delta}-\boldsymbol{B}_{\boldsymbol{C M F - 1}}$ at center of slit-1 and $\boldsymbol{\delta}-\boldsymbol{B}_{\boldsymbol{C M F - 2}}$ at slit-2, then electron-21 with $\boldsymbol{C M F}$ impact on the screen together with waves from 1 to 20 . An electron enters only one slit with stronger $\boldsymbol{C M F}$, while electron's weaker $\boldsymbol{C M F}$ enters the other slit; depending on $r_{\mathrm{mn}}, \boldsymbol{C M F - 2}$ at slots-2 range from $0.25 \%$ to $56.25 \%$ of $\boldsymbol{C M F - 1}$ at slot-1.

As electrons emerged from electron gun, each produced CMF or $B_{C M F}$ along its path, while surrounded by an Electric Field (E-F), shown in two dimension for electrons-26-25 and 24 with magenta color, moving to either slits, the cross sectional plan of electron-23 is shown with CMF and the E-F extended from its center to all sides while approaching slit-2, the CMF $\left(B_{C M F}\right)$ intensity is proportional to the velocity as given by Eq. (1), and to the acceleration potential as given by Eq. (15), thus an electron entered the slit; as shown for electron-22 at the center of slit- 1 together with its strongest $\delta-B_{C M F-1}$ this occurred concurrently with other less magnitude portion of its extension $\delta-B_{C M F-2}$ at the center of slit-2, their existence is similar to both $\delta$-Wave entering both slits in Young's double slit experiment shown in Fig.2-D, since electron velocity is given in terms of energy by Eq. (12), therefore substituting $V_{e}$ from Eq. (12) with $V_{e}$ in Eq. (1) then rearranging the equation, the CMF $\left(B_{C M F}\right)$ entering slit-1 due to specific energy is given by

$$
\frac{B_{C M F-1}^{2} r_{m}^{4} c^{2}}{q^{2}}=\frac{2 e E}{m}
$$

Therefore, from Eq. (21), the magnitude of the $\boldsymbol{C M F - 1}\left(B_{C M F-1}\right)$ entered a slit with an electron (electron-22 at slit-1 in Fig.3), is given by

$$
B_{C M F-1}=\sqrt{\frac{2 \mathrm{E} q^{2}}{m r_{m}^{4} c^{2}}} \quad T
$$

Where, $r_{m}$ is the magnetic radius for $B_{C M F-1}$ measured from electron' center to edge of slit-1, the $\boldsymbol{C M F}-2$ $\left(B_{C M F-2}\right)$ entering a slit without an electron (slit-2 in Fig.3), is given by

$$
B_{C M F-2}=\sqrt{\frac{2 \mathrm{E} q^{2}}{m r_{m n}^{4} c^{2}}} T
$$

Where, $r_{m n}$ is the magnetic radius for $B_{C M F-2}$ measured from electron center to the center of slit-2, it is weaker (by $r_{m n}-r_{m}$ ) than $B_{C M F-1}$ entering slit-1, since a recent double slits experiment suggested that each electron somehow travels through both slits at the same time and interferes with itself, like a wave instead of a particle, resulted in interference pattern [51], therefore, the existence in Fig.3, of $\delta-\boldsymbol{C M F}-1\left(B_{C M F-1}\right)$ in the canter of slit-1 and $\boldsymbol{\delta}-\boldsymbol{C M F - 2}\left(B_{C M F-2}\right)$ in the center of slit-2, is similar to the existence in Fig. 2 -D of $\delta$-Wavelet1 in the canter of slit- 1 and $\delta$-Wavelet- 2 in the center of slit-2, and since $\delta$-Wavelet- 1 and $\delta$-Wavelet- 2 are part of the EM-R been transformed into $\boldsymbol{P W}\left(B_{P W}\right)$ through the polarization process given by Eq. (19), after emerged from both slit-1 and slit-2 respectively, therefore the $\delta$-Wavelet-1 and $\delta$-Wavelet- 2 in Fig. 3 are the $\boldsymbol{C M F}$ $\left(B_{C M F}\right)$, and both $\boldsymbol{C M F}$ represents the same electron producing them, but with slightly different magnitudes, hence both $\boldsymbol{C M F}\left(B_{C M F}\right)$ are wrongly perceived.

As both experiments shown in Fig.2 and Fig.3, are characterized in common with $\delta-B_{C M F}$ entering both slits, and since both having $\delta-B_{C M F}$ transformed into $\boldsymbol{P W}\left(B_{P W}\right)$ and $\boldsymbol{C M F}\left(B_{C M F}\right)$ respectively, after emerging from the two experiments slits, therefore both $\boldsymbol{P W}\left(B_{P W}\right)$ and $\boldsymbol{C M F}\left(B_{C M F}\right)$ formed successive trail of progressive enlarged waves, hence both waves caused interference, adding and cancelling each other to form the bright fringe or patches showing in Fig.3, similar to Young's double slit experiment in Fig.2, but this has been perceived and interpreted as waves collapse back into a single particle on screen, which is the foundation problem of quantum mechanics [52], and as shown there is no magic in this process.

The magnitude of $\boldsymbol{C M F}\left(B_{C M F}\right)$ in EM-R, entering any slit in Fig.2 is given by Eq. (9), and for electron-CMF and related $\boldsymbol{C M F}$ entering slit-1\&2 in Fig.3, it is derived using Eqs. (22\&23) an examples of which is given in Table.3, while the equivalent frequency if both $\delta \mathbf{C M F}-\mathbf{1}\left(B_{C M F-1}\right)$ and $\boldsymbol{\delta}-\boldsymbol{C M F}-\mathbf{2}\left(B_{C M F-2}\right)$ at slit-1 and slit-2 in Fig.3, or both $\boldsymbol{\delta}$-CMF-1 $\left(B_{C M F-1}\right)$ and $\boldsymbol{\delta}-\boldsymbol{C M F - 2}\left(B_{C M F-2}\right)$ in Fig.2, are transformed into EM-R, is derived from Eq. (9), and given by

$$
v=\sqrt[5]{\frac{B_{C M F}^{2}}{C_{B}}} \quad H z
$$

The equivalent frequency given by Eq. (24), showed that for a $\boldsymbol{C M F}$ wave accompanying an electron to produced intensity similar to EM-R, when detected at the monitoring in Fig.3, a minimum intensity is required 
(can be tested from Table.1), hence this is done by controlling electron velocity or the accelerator potential, the equivalence wavelength of $B_{C M F}$ in Eqs. $(9 \& 22)$ is given as

$$
v^{5}=\frac{2 \mathrm{E} q^{2}}{C_{B} m r_{m}^{4} c^{2}}
$$

Substituting $v$ with $\frac{c}{\lambda}$ in Eq. (25), hence the wavelength is given by

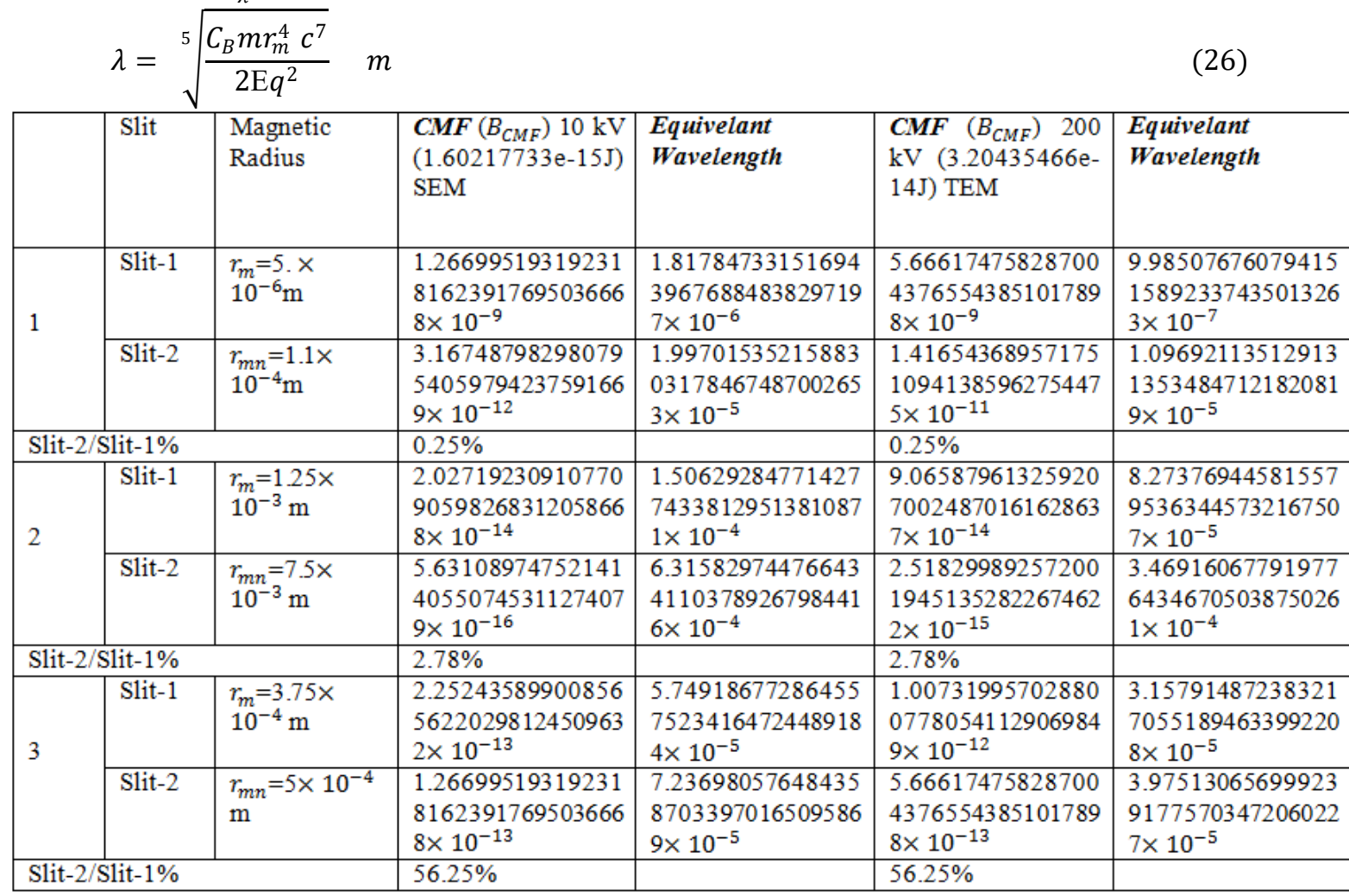

Table.3. The relative magnitudes of Circular Magnetic Field One $\left(B_{C M F-1}\right)$ and Two $\left(B_{C M F-2}\right)$ entering slitand slit-2, respectively in Fig.3, using Eqs. (22\&23) with acceleration potentials of $10 \mathrm{kV}$ and $200 \mathrm{kV}$ respectively, in (1) $r_{\mathrm{m}}=5 \times 10^{-6}$ and $\mathrm{r}_{\mathrm{mn}}=1 \times 10^{-4}$ [53], while (2\&3) are mixture of $r_{m n}=5 \times 10^{-3} \mathrm{~mm}$ [44].

The Eq. (26) can be simplified, and given by

$$
\lambda=\sqrt[5]{\frac{5.088825984 \times 10^{-23} r_{m}^{4}}{\mathrm{E}}} \mathrm{m}
$$

Since $r_{\mathrm{m}}=\frac{\lambda}{4}$, substituting this in Eq. (26), hence when energy is given, the equivalent wavelength is given by

$$
\lambda=\frac{C_{B} m c^{7}}{4^{4} 2 \mathrm{E} q^{2}} \quad m
$$

Or for simplicity as

$$
\lambda=\frac{1.98782265 \times 10^{-25}}{\mathrm{E}} \quad m
$$

\section{The Nature Of Plank's Constant}

Equation (29) can reproduce the wavelength versus particle energy for electrons given by Kittel [54], and since the wavelength equal four magnetic radius $\left(\lambda=4 r_{\mathrm{m}}\right)$ substituting this in Eq. (28), the Radiation Energy $\left(E_{R}\right)$ is given by

$$
E_{R}=\frac{C_{B} m c^{7}}{2048 q^{2} r_{\mathrm{m}}} \quad J
$$

For simplicity, the Radiation Energy $\left(E_{R}\right)$ can also be given by

$$
E_{R}=\frac{4.969556625 \times 10^{-26}}{r_{\mathrm{m}}} \quad J
$$

Substituting $\lambda$ with $\frac{c}{f}$ in Eq. (28), when energy is known, then frequency is given by 


$$
v=\frac{2\left(4^{4}\right) E_{R} q^{2}}{C_{B} m c^{6}} \quad H z
$$

Solving the fixed parameters and radiation constant $C_{R}$, therefore Eq. (32) can be written as

$$
v=1.5091889610977116092323427343983 \times 10^{33} E_{R} \quad H z
$$

From Eq. (33), knowing the frequency $v$, the energy of any EM-R is given by

$$
E_{R}=\frac{v}{1.5091889610977116092323427343983 \times 10^{33}} \quad J
$$

But the inverse of $1.5091889610977116092323427343983 \times 10^{33}$ in Eq. (34) is the Planck' formula

$$
E_{R}=6.6260755 \times 10^{-34} v=h v \quad J
$$

Alternatively, replacing $E_{R}$ in Eq. (34) with Planck' energy formula, the following is obtained

$$
h v=\frac{v}{1.5091889610977116092323427343983 \times 10^{33}} \quad J
$$

Cancelling the frequency $v$ from both sides of Eq. (36), therefore, the given Planck constant [16], is also given by

$$
h=\frac{1}{1.5091889610977116092323427343983 \times 10^{33}} \quad J . s
$$

Therefore the inverse of Eq. (37), is the Planck' constant

$$
h=6.6260755 \times 10^{-34} \quad J . S
$$

But what is the nature of this Planck' constant? Going to Eq. (8), the Planck constant is given by

$$
h=\frac{B_{C M F}^{2} m_{e} c^{6}}{2\left(4^{4}\right) q^{2} v^{5}} \quad J . s
$$

During energy production, the frequency is not the main factor, rather it is the time $\left(t_{F}\right)$ [25], and since $t_{F}=\frac{1}{v}$, hence Eq.(39) becomes

$$
h=\frac{B_{C M F}^{2} t_{F}^{5} m_{e} c^{6}}{2\left(4^{4}\right) q^{2}} \quad J . S
$$

Where, $t_{F}$ is Flipping time in second, but electron's mass (m), charge (q) and speed of light (c) in Eq. (40) has fixed quantity, the only variables are the CMF $\left(B_{C M F}\right)$ and the Flipping Time $\left(t_{F}\right)$, and the variation of both quantities $\left(B_{C M F}\right.$ and $\left.t_{F}\right)$ in Eq. (40) as given in Table.1, is in a manner to keep the Planck' constant at fixed magnitude, the products of both the $\mathrm{CMF}\left(B_{C M F}\right)$ and time $\left(t_{F}\right)$ in Eq. (40) is given by

$$
B_{C M F}^{2} t_{F}^{5}=\frac{h}{5.0527252584917691102101768251341 \times 10^{55}}=\frac{h}{C_{F}}
$$

Where, 5.0527252584917691102101768251341 $\times 10^{55}$ is the Fixed constant $\left(C_{F}\right)$, from Eq. (41), the magnitude of this product is given by

$$
B_{C M F}^{2} t_{F}^{5}=1.3113864619620884691409896280354 \times 10^{-89}=\left(C_{R}\right)
$$

But the value $1.3113864619620884691409896280354 \times 10^{-89}$ is the radiation constant $C_{R}$ given in Eq. (9), with related data in Table.1, hence Eq. (42) shows that, the multiplication of both the CMF $\left(B_{C M F}\right)$ and the Flipping time $\left(t_{F}\right)$ produced constant value for any EM-R wave, therefore from Eqs. (41\&42) the Plank' constant represents the following two constants

$$
h=C_{R} C_{F} \quad \text { J.S }
$$

Therefore, the multiplication of the Fixed constant $\left(C_{F}\right)$ in Eq. (41) by radiation constant $\left(C_{R}\right)$ given by multiplication of both CMF $\left(B_{C M F}\right)$ and the Flipping time $\left(t_{F}\right)$, for any of their value in Table.1, produced the Planck' constant $h$, therefore Planck' constant existed in the combined value of both the CMF $\left(B_{C M F}\right)$ and the Flipping time $\left(t_{F}\right)$, it only emerged when Fixed constant $\left(C_{F}\right)$ is multiplied by radiation constant $\left(C_{R}\right)$ as given in Eq. (43), therefore, the origin of Planck' constant can be expressed as

$$
h=B_{C M F}^{2} t_{F}^{5} C_{F} \quad \text { J.s }
$$

Therefore, the Planck' constant can also be given by

$$
h=B_{C M F}^{2} t_{F}^{5} 5.0527252584917691102101768251341 \times 10^{55} \quad \text { J.s }
$$

Multiplying both part of Eq. (45) by frequency $v$, the Radiation Energy $\left(E_{R}\right)$ is given by

$$
E_{R}=h v=\left(B_{C M F}^{2} t^{5} 5.0527252584917691102101768251341 \times 10^{55}\right) v \quad J \quad \text { (46) }
$$

Changing the frequency $(v)$ in Eq. (46) with time $\left(t_{F}\right)$, therefore the Radiation Energy $\left(E_{R}\right)$ is given by

$$
E_{R}=h v=B_{C M F}^{2} t_{F}^{4} 5.0527252584917691102101768251341 \times 10^{55} \mathrm{~J}
$$

This radiation energy can be expressed by

$$
E_{R}=h v=C_{R} C_{F} v \quad J . S
$$

\section{Results And Discussion}

This paper is a modified version of the "Double Slit Experiment-Explained" [21], which the publisher failed to replace, regardless of written promised. 
In this paper, suggestions are made regarding relation between Electromagnetic Radiation (EM-R) entering and emerging from small hole or slits:

When enter and emerge from slit/hole/biaxial crystal, the EM-R lost the electric field due to polarization effect.

The wavelet which entered and emerged from slits is the magnetic part of the EM-R.

When emerged from the hole or slit this magnetic wave becomes circular in shape.

The resulted Conical Diffraction (CD), lost characteristics of EM-R radiation, particularly the speed of light, as electric field is lost.

The shape of a diffracted wave on screen due to a single slit/hole/biaxial crystal is representation of the emerged $\boldsymbol{C D}$ or $(\boldsymbol{C M F})$, while $\boldsymbol{C D}$ waves from two slits interfere constructively or destructively.

The usage of slit to polarize EM-R, will never yield the result as the biaxial crystal, which has very small radius comparable to the wavelength of the wave, therefore it is the reason why the practical requirements for narrow slits which are the source of just one Huygens' wavelet are difficult if not impossible to achieve [44], while its rather easy in the biaxial crystal [28].

The shape of CD in Fig.2-D using slit [42] is similar to CD using biaxial crystal [28].

The double-slit experiment, or the electron diffraction, is described as the entre of $\boldsymbol{C M F}$ from single electron into two slits, with different magnitudes, the $\boldsymbol{C M F}$ which accompanied by the electron through a slit has the greater magnitude.

The ceased of interference pattern when detector is put near one of the slits to determine which slit(s) an electron is passing through, at which electrons create two straight lines, like classical particles [50], as shown in Fig.3, such detection interfere with the electrons $\boldsymbol{C M F}$ and passing through both slits gives the lines.

As showed by Eq. (1), the $\boldsymbol{C M F}$ produced by such electron is the main energy of electromagnetic radiation wave, therefore the so-called phase waves or matter waves or plot wave which exhibit certain striking points of similarity with electromagnetic waves, particularly in their ability to produce the diffraction effects by which they were discovered [12], is just a $\boldsymbol{C M F}$.

G.P. Thomson was correct right from the beginning in realizing de Broglie's theory as a theory of light and electronic orbits, not as a theory of electron diffraction [24].

Planck' constant is related to the double slit experiment by the energetic $\boldsymbol{C M F}$ and relation with energy production.

As shown in Eq. (43) Planck' constant consists of two constants, the fixed (m, q and c) parameters of the energy formula designated as Fixed Constant $\left(C_{F}\right)$ and the two variables the CMF $\left(B_{C M F}\right)$ and the Flipping time $\left(t_{F}\right)$ designated as Radiation Constant $\left(C_{R}\right)$.

The multiplication of Eq. (47) by $t^{4}$ gives the radiation energy, while multiplication of the same equation be $t^{5}$ gives the Planck' constant, as in Eq. (44).

Therefore, Planck' constant is the variation of Radiation Constant $\left(C_{R}\right)$, which contains both the $\mathrm{CMF}\left(B_{C M F}\right)$ and the Flipping time $\left(t_{F}\right)$, the multiplication of which gives a constant value for each radiation, and it's multiplication by the Fixed Constant parameters $\left(C_{F}\right)$ produced the Planck' constant $(h)$.

It is clear from Eq. (44), that Planck was correct in stating that; "his constant is merely a mathematical trick to obtain the right description (formula) of the black body radiation spectral intensity profile." [55]

\section{Conclusion}

The disclosed knowledge of the Magnetic Force $\left(F_{\mathrm{m}}\right)$ [19], Spinning Magnetic Field (SMF), and the produced Spinning Magnetic Force (SMFc) [20], allowed the Flip-Flop (F-F) mechanism for Electromagnetic Radiation (EM-R) [25], and demonstrate the Radiation Magnetic Force $\left(F_{m R}\right)$ with similarity in nature to Planck' energy formula $(h v)$ as an embedded in EM-R, thus excluding quanta (photon) in removing electron from atom in Photoelectric Effect [16], the F-F condition and parameters for the speed of light was derived [26], showed Compton Effect as an internal production of Secondary EM-R (S-EM-R), with the existence of Electromagnetic Radiation Force $\left(F_{E M R}\right)$, pulling the produced EM-Wave $(E M-W)$ at the ends of F-F mechanism [18]. These background lead to the suggestion that, the diffraction phenomenon is due to a change in characteristics of EM-R, resulted in Conical Diffraction (CD), similar in nature and characteristics to the CD emerged from the biaxial crystal [28], the CD is a wave neither travel with speed of light, nor carrying electric field, the wave is not semi-circle, rather it is a full circular wave, and composed of the magnetic part of the EMR, or the Circular Magnetic Field (CMF), originated from accelerated electrons [25] given by Eq.(1), therefore the passage of light through a single hole/slit/biaxial crystal resulted in rings on the monitor, while the interference of two such $\boldsymbol{C D}$ in double slits experiment, produced constructive or destructive interference, shown as patches on the screen. Thus electron diffraction in double slit experiment is interpreted as the entry and emerge of $\boldsymbol{C M F}$ in and out of both slits/holes in addition to the electron producing them, thus both $\boldsymbol{C M F}$ produced constructive or destructive interference; while the origin of the Planck' constant $(h)$ is finally derived, and showed to form relationship between two constants, the Radiation constant $\left(C_{R}\right)$, composed of both the 
CMF $\left(B_{C M F}\right)$ and the Flipping time $\left(t_{F}\right)$, and the Fixed constant $\left(C_{F}\right)$, the Planck' constant resulted from the multiplication of both constants, hence a mathematical trick.

Finally, G.P. Thomson found it impossible to explain his results "except by the assumption of some kind of diffraction" [11, 24], simply because he faced what Compton faced before [5], contrary to Compton, and all of Davisson/Germer and Thomson, who followed Einstein quanta (photon) line [5, 9] [11], Raman understood the problem by early stating that "the classical wave-principles are not easily reconcilable with Compton effect because they have not been correctly interpreted," [56]; the irrelevant simplistic explanation of billiard-ball of quanta, allowed the emergence of such complex ideas and alleged predication by Quantum Mechanics $(\mathrm{QM})$ that any detector capable of determining the path taken by a particle through one or the other of a two-slit plate will destroy the interference pattern [57], such line of thoughts empowered some to think QM represents the super knowledge, even an attempt has been made to establish relation between it and higher brain functions [58], leading some to imagine QM as a steppingstone between ourselves and the Universe, between what we want and making it actually happen in the natural [59]; hence what QM succeeded to attained was to get rid of common sense because as it claimed common sense makes a lot of mistaken assumptions [13].

Finally, Feynman once described the double-slit experiment, stating that, "we choose to examine a phenomenon which is impossible, absolutely impossible, to explain in any classical way, and which is in the heart of quantum mechanics" [60], but as seen some great historical lessons could be draw from this experience that, the collection of lots of data without being able to find any basic underlying principles is not science [27], closure of any scientific debate on alleged accomplishment is not scientific in nature, and science is an open field, in which an exploration by an individual could benefit and progress humankind.

\section{References}

[1]. A Einstein \& into English T, Concerning an Heuristic point of view toward the emission and transformation of light, American Journal of Physics, vol. 33, 1965.

[2]. D Finkelstein, What is a photon? OPN trends, supplement to Optics \& Photonics News Vol. 14. No. 10, 2003.

[3]. R A Millikan, A Direct Photoelectric Determination of Planck's “h” Physical Review, vol. 7, 1916.

[4]. R. H. Stuewer, Einstein's Revolutionary Light-Quantum Hypothesis, Christian Joas, Christoph Lehner, and Jürgen Renn (eds.), Conference on the History of Quantum Physics, Max Planck Institute for the History of Science, 2008.

[5]. A H Compton, "A Quantum Theory of the Scattering of X-Rays by Light Elements", Physical Review, vol. $21,1923$.

[6]. M. Sachs, Einstein versus Bohr, Open Court, 1988

[7]. L. Marion, H. M. Van, Electron Diffraction, Encyclopedia of Physics, Ed Lerner, R. G. and Trigg, G. L., VCH Publishers, Inc., 303-305, 1991

[8]. L. de Broglie, The wave nature of the electron Nobel Lecture, 1929

[9]. C J Davisson, L. Germer H Diffraction of Electrons by a Crystal of Nickel, Phys. Rev. 30, 705, 1927.

[10]. Advanced Physics Laboratory, Electron Diffraction and Crystal structure, University of Michigan, 2006.

[11]. G. P. Thomson (1928) Proc. Roy. Soc. 117, 600.

[12]. Harnwell, Livinggood Experimental Atomic Physics, McGraw-Hill, 1961.

[13]. L N Hoang, The Essence of Quantum Mechanics, Science 4 All, 2013.

[14]. L. L. Williams, The Spirit of Reason, Online Edition, KONFLUENCE PRESS Manitou Springs, Colorado, 2006.

[15]. M Shih, Developing Ideas about Photons:(since the First Paper about Photoelectric Effect by Einstein in 1905).. AAAPPS Bulletin, $15(1), 2005$

[16]. M E Yousif The Photoelectric Effects-Radiation Based With Atomic Model, International Journal of Fundamental Physical Sciences (IJFPS), vol. 5, 2015.

[17]. M Planck, Annalen der Physik 4(553), 1, 1901.

[18]. M E Yousif, The Compton Effect Re-Visited, J Adv Appl Phys, 1:004, 2016

[19]. M E Yousif, The Magnetic Interaction, Comprehensive Theory Articles, Journal of Theoretics, vol. 5, 2003a.

[20]. M E Yousif, THE SPINNING MAGNETIC FORCE, Comprehensive Theory Articles, Journal of Theoretics, vol. 5, 2003b.

[21]. M E Yousif, The Double Slit Experiment-Explained. J Phys Math 7: 179. doi:10.4172/2090-0902.1000179, 2016.

[22]. Discussion with Dr. Kai Fauth of University of Wuerzburg, at researchgate.net, 2013.

[23]. D Cassidy, G. Holton, J. Rutherford, Understanding Physics, Springer-Verlag, New York, 2002.

[24]. J. Navarro, Planck and de Broglie in the Thomson Family, Christian Joas, Christoph Lehner, and Jürgen Renn (eds.), Conference on the History of Quantum Physics, Max Planck Institute for the History of Science, 2008.

[25]. M E Yousif, The Electromagnetic Radiation Mechanism, International Journal of Fundamental Physical Sciences (IJFPS), vol. 4, $2014 \mathrm{a}$.

[26]. M E Yousif, Electromagnetic Radiation Energy and Planck' Constant, International Journal of Innovative Research in Advanced Engineering (IJIRAE), vol. 1, 2014b.

[27]. B Crowell, Light and Matter, Benjamin Crowell, 1998.

[28]. M. V. BERRY, M. R. JEFFREY, J. G. LUNNEY, Conical diffraction: observations and theory, Proceeding of the Royal Society, doi:10.1098/rspa.2006.1680, Proc. R. Soc. A 462, 1629-1642, 2006.

[29]. K Kalkandjiev, Maria AB Conical refraction: an experimental introduction, Photon Management III, edited by John Sheridan, Frank Wyrowski Proc. SPIE Vol. 6994, 69940B, 0277-786X/08//\$18 · doi: 10.1117/12.780793, 2008.

[30]. R. Beeching, Electron Diffraction, Methuen \& Co. Ltd. 36 Essex Street, W.C. London, 1936.

[31]. J B Hastings, F. M. Rudakov, D. H. Dowell, J. F. Schmerge, J. D. Cardoza, J. M. Castro, S. M. Gierman, H. Loos, P. M. Weber, ULTRAFAST TIME-RESOLVE D ELECTRON DIFFRACTION WITH MEGAVOLT ELECTRON BEAMS, Applied Physics Letters, SLAC-PUB-12162, 2006.

[32]. W. L. BRAGG, The Diffraction of Short Electromagnetic Waves by a Crystal, Proc. Camb. Phil. Soc., XVII (I), 1912.

[33]. M E Yousif, Interaction of Intense Magnetic Fields with Crystals, Unpublished.

[34]. Wikipedia, Deferent and epicycle, From Wikipedia, the free encyclopedia, 17 June 2015.

[35]. E. Nightingale, Magnetism and Electricity, G. Bell and Sons Ltd, London, 1958. 
[36]. F. E. Trinklein, Modern Physics, Holt, Rinehart and Winston Inc., New York, 1990

[37]. L. Novotny, Lecture Notes on ELECTROMAGNETIC FIELDS AND WAVES, ETH Zurich, Photonics Laboratory, 2014.

[38]. J. Newman, Physics of the life sciences: Springer, 2008.

[39]. M. Alonso, E. J. Finn, Fundamental University Physics V. II Field and Waves, Addison and Wesley, Massachusetts, 1967.

[40]. J. R. Ballif, Conceptual Physics, Wiley N. Y., 1969.

[41]. W. R. Fuch, Modern Physics Weidenfield \& Nicolson (Educational) Ltd: and The Macmillan for Translation, Zurich, 1967.

[42]. T. Tajima, G. Mourou, A Recent Development in High Field Science, K. Itakura, et al., (edited) "Proceedings of International Conference on Physics in Intense Fields, PIF, 2010a.

[43]. K. Itakura, Strong Field Dynamics in Heavy Ion Collisions, K. Itakura, et al., (edited) "Proceedings of International Conference on Physics in Intense Fields (PIF2010)," edited by K. Itakura, et al, 2010b.

[44]. C. Hammond, The basics of crystallography and diffraction (third edirion), Institute for Materials Research, University of Leeds, INTERNATIONAL UNION OF CRYSTALLOGRAPHY, Oxford University Press, 2009.

[45]. J E Ebner, Helical Electromagnetic Waves, The General Science Journal, 2012.

[46]. Wikipedia.org, Polarizer, 2014a.

[47]. M. M. Ripoll, "Crystallography-Cristalografia", Dept. of Crystallography \& Struc. Spanish National Research Council, Madrid, 2016.

[48]. Wikipedia, Arago spot, 2015.

[49]. C V Raman, The phenomena of conical refraction, Curr. Sci. 11, 44-46, 1942

[50]. A D Cronin, Jörg Schmiedmayer, and David E. Pritchard, Optics and interferometry with atoms and molecules, REVIEWS OF MODERN PHYSICS, Vol. 81, 2009.

[51]. L. Zyga, Which-way detector unlocks some mystery of the double-slit experiment, Phys.org, 2011.

[52]. Vacuum http://www.vacuum-mechanics.com/

[53]. Physics 50 Webpage, 37 Diffraction, Spring, 2010.

[54]. C. Kittel, Introduction to Solid-State Physics, John Wiley \& Sons, Inc, 1957.

[55]. P C Deshmukh and V Shyamala, 100 Years of Einstein's Photoelectric Effect, Wednesday, 2006.

[56]. C V Raman, A classical derivation of the Compton Effect, Indian J. Phys, vol. 3, 1928

[57]. R Mario, EXAMINATION OF WAVE-PARTICLE DUALITY VIA TWO-SLIT INTERFERENCE, arXiv:physics/0302062 [physics.gen-ph]

[58]. C. Koch, K. Hepp, The relation between quantum mechanics and higher brain functions: Lessons from quantum computation and neurobiology, caltech.edu, 2007.

[59]. P. Baksa, Can Quantum Physics Explain God? Huffingtonpost.com, 2014

[60]. R. P. Feynman, R. B. Leighton, and M. Sands, The Feynman Lectures in Physics Volume 3, Section 1-1, Addison-Wesley, 1965. 